\title{
Filaggrin mutations are associated with ichthyosis vulgaris in the Southern Chinese population*
}

\author{
Chang-Xing $\mathrm{Li}^{1}$, Quan Luo ${ }^{2}$, Xue-Mei Li ${ }^{2}$, Xi-Bao Zhang ${ }^{2 \#}$, Chun-Lei Han ${ }^{1}$, Ze-Lin Ma ${ }^{1}$, \\ Dong-Zi Lin ${ }^{1}$
}

\footnotetext{
${ }^{1}$ Department of Dermatology, Dongguan Institute of Dermatology, Dongguan, China;

${ }^{2}$ Department of Dermatology, Guangzhou Institute of Dermatology, Guangzhou, China;

${ }^{\#}$ Corresponding Author: lilichangxing@163.com;

Authors Chang-Xing Li, and Quan Luo contributed equally to this work.
}

Received 13 October 2010; revised 1 November 2010; accepted 5 November 2010

\begin{abstract}
Filaggrin (FLG) plays an important role in the epidermal barrier function, which identified in patients with ichthyosis vulgaris(IV).To study the genetics of FLG mutations in Southern Chinese patients with IV. We evaluated the influence of five mutations (3321 delA, 441delA, 1249 insG, E1795X and S3296X) in a cohort of 65 IV Chinese patients and in 100 control individuals using the Sequenom ${ }^{\circledR}$ MassARRAY ${ }^{\circledR}$ system. The null allele frequency of 3321delA was $52.31 \%(34 / 65)$. FLG mutation 441delA was only found in one IV patients. FLG mutations 1249insG, E1795X and S3296X were not found in these patients. These findings show that the mutation 3321delA represent the most frequent genetic cause in Southern Chinese IV patients. Our findings confirm and extend the knowledge of the influence of FLG mutations in IV.
\end{abstract}

Keywords: Ichthyosis Vulgaris; Southern Chinese Population; Filaggrin; Mutation

\section{INTRODUCTION}

Ichthyosis vulgaris (OMIM 146700) is the most common ichthyosis with an estimated prevalence of 1:2501000 , and inherited in a semidominant pattern [1]. Patients have light grey scaling, keratosis pilaris, increased palmoplantar markings and associated atopic manifestations. Involvement is generally mild and may vary greatly with climate and humidity. There is reduced or absence of keratohyalin granules in the epidermis [2]. In addition, there is a decrease or absence of profilaggrin, a major component of keratohyalin granules $[3,4]$.

\footnotetext{
*Supported by Medical Scientific Research Foundation of Guangdong Province, China
}

FLG is coding gene located in the epidermal differentiation complex on chromosome 1q21 [5]. Recently, mutations in the FLG have been shown to be a major risk factor for IV and atopic dermatitis(AD) [5,6]. The association of FLG mutations with IV in Northern European, Japanese, Bangladeshi, Korean, Taiwanese and Singaporean Chinese populations has been confirmed in subsequent studies [7-17]. To date, more than different $20 \mathrm{mu}-$ tations have been identified in different populations, all leading to a filaggrin deficiency. FLG mutations 3321delA, 441delA, 1249insG, E1795X and S3296X have been previously reported, from Asia including China, Japan, Korea, Taiwan [8]. In this study, we identified five FLG mutations 3321delA, 441delA, 1249insG, E1795X and S3296X in Southern Chinese IV patients.

\section{MATERIALS AND METHODS}

\subsection{Cases and Controls}

DNA samples from 65 IV were of Southern China origin and recruited with ethical approval and had given their informed consent. The study was conducted according to the principles of the Declaration of Helsinki and all applicable local regulations related to protection of human subjects. The institutional review boards of the respective institutions approved the study. DNA samples from 100 healthy volunteers were available to serve as controls. Controls had never been diagnosed with IV or AD and information from medical records where available.

\subsection{Genotyping}

Genomic DNA was extracted from peripheral blood using standard techniques. All SNPs were genotyped by MassARRAYTM system (Sequenom, San Diego, CA, http://www.sequenom.com/ ). The iPLEX ${ }^{\mathrm{TM}}$ assay was followed according to manufacturers instructions (http:// www.sequenom.com/) using $10 \mathrm{ng}$ of genomic DNA. The primer sequences are available upon request (primer sequences in Table 1). 
Table 1. Primer sequences for all 5 FLG mutations using iPlex chemistry on a MassARRAY platform.

\begin{tabular}{|c|c|c|c|}
\hline Mutations & $\begin{array}{l}\text { Polymerase chain reaction primer } 1 \\
\text { sequence }\end{array}$ & $\begin{array}{l}\text { Polymerase chain reaction primer } 2 \\
\text { sequence }\end{array}$ & $\begin{array}{lll}\begin{array}{l}\text { Extension } \\
\text { quence }\end{array} & \text { primer se- } \\
\end{array}$ \\
\hline$E 1795 X$ & $\mathrm{~A}$ & $3^{\prime}$ & TCTCGTGCCTGCT \\
\hline 1249 ins $G$ & $\begin{array}{l}\text { TGGATGGGCAAGCTTCATC } \\
\text { C-3, }\end{array}$ & $\begin{array}{l}\text { ITGTGAATGTCCCT } \\
3,\end{array}$ & $\begin{array}{l}\text { 5'-GCCTGACTACCGCT } \\
\text { AG-3' }\end{array}$ \\
\hline $441 \operatorname{del} A$ & $\begin{array}{l}\text { GGATGCCCTCAAGTCTG } \\
\text { AG-3, }\end{array}$ & TGTTCAGAACTAG & $\begin{array}{l}\text { CATGCCTTTTCCCC } \\
\text {, }\end{array}$ \\
\hline S3296X & $\begin{array}{l}\text { 5'-ACGTTGGATGTGCATCATCCCAT } \\
\text { GAACAGG-3' }\end{array}$ & $\begin{array}{l}\text { 5'-ACGTTGGATGTGTCTGCTGAC } \\
\text { TGCTGGTG-3' }\end{array}$ & $\begin{array}{l}\text { 5'-GTGTCTCTCTCCTGG } \\
\text { ACTT-3' }\end{array}$ \\
\hline
\end{tabular}

\subsection{Statistical Analysis}

Genotype frequencies between cases and controls were using the continuity-adjusted chi-square test. The reported P-values are the results of two-sided tests. P-values $\leq 0.05$ were considered to be statistically significant. All computations have been performed using SAS software version 8.01.

\section{RESULTS}

A total of 5 polymorphic SNPs across the FLG gene locus were analysed (Figures 1,2). The minor allele frequencies of the two functional polymorphisms $(7638 \mathrm{G} / \mathrm{G}$ and $7717.9 \mathrm{~A} / \mathrm{A}$ ) located in the FLG gene were $52.31 \%$ and $1.54 \%$ in the IV cases and $5.17 \%$ and $0 \%$ in controls, respectively. The assay showed good clusters and mass spectra (Figures 1,2). The null allele frequency of FLG mutation $3321 \mathrm{delA}$ were $52.31 \%(34 / 65)$ and $4 \%(4 / 100)$ in cases and controls respectively. FLG mutation 441 delA was only found in one IV patients. FLG mutation 441delA was not found in the controls. FLG muta- tions 1249insG, E1795X and S3296X were not found in these patients and controls. When comparing IV patients with controls, only 3321delA mutation showed a statistically significant association with IV $\left(\chi^{2}=49.1718 \mathrm{P}<\right.$ $0.0001)$.

\section{DISCUSSION}

IV and AD are relatively common heritable skin diseases. Lack of expression of the protein filaggrin has been shown to predispose to the development of IV and atopic eczema or dermatitis [5,16]. The filaggrin gene resides on human chromosome 1q21 within the epidermal differentiation complex, a region that also harbours genes for several other proteins that are important for the normal barrier function of the epidermis [5].

Recent studies showed that both diseases are closely related to loss-of-function mutations in the FLG. The homozygous or compound heterozygous loss-of-function mutations p.R501X and c.2282del4 in FLG have been identified in moderate or severe IV patients from Ireland,
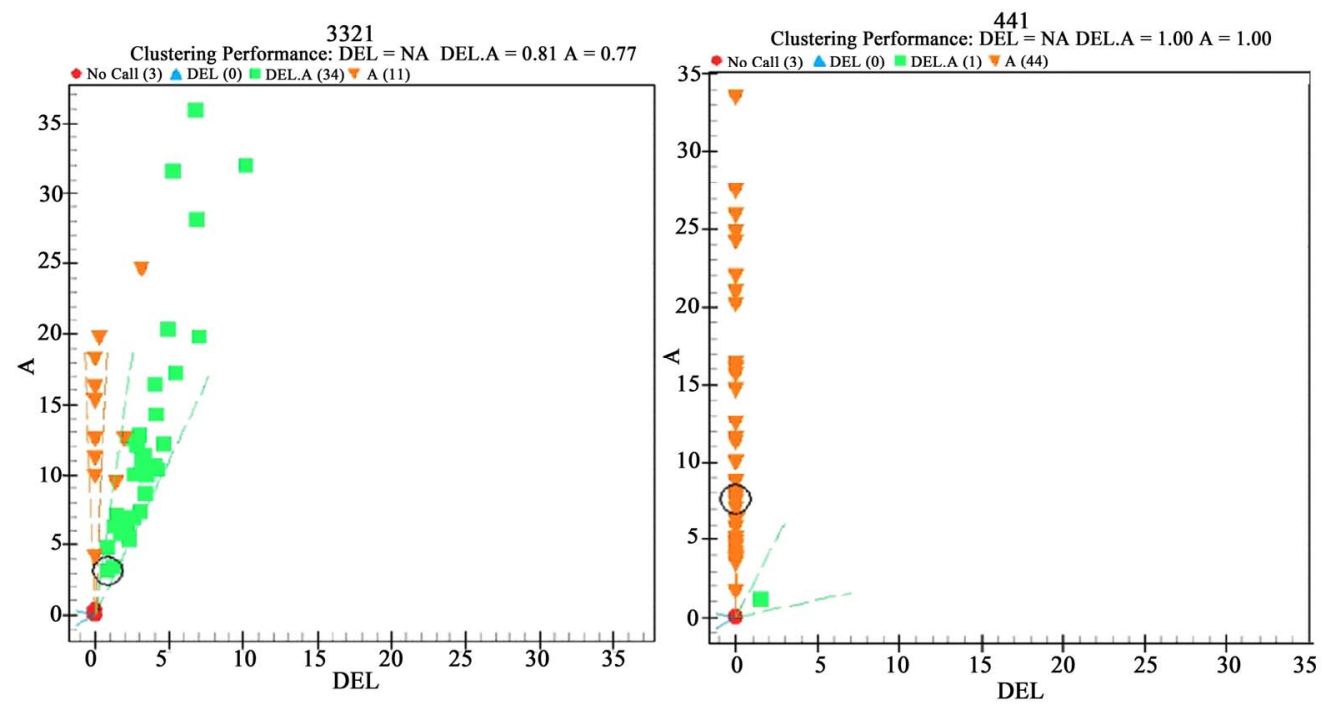

Figure 1. FLG mutation $3321 \mathrm{del}$ and 441delA genotyped in 65 IV cases and 100 controls using Sequenom MassARRAY platform. The assay showed good clusters. No obvious genotyping errors were apparent. 


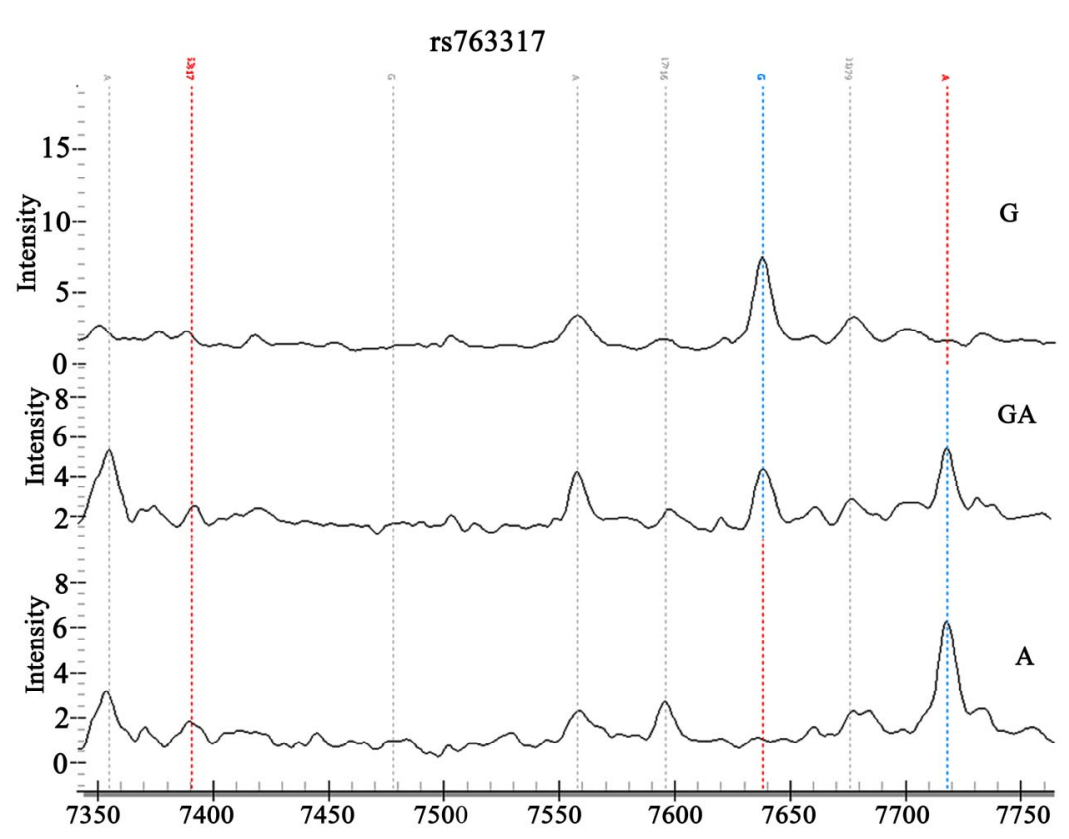

Figure 2. FLG mutation 3321 del and 441delA genotyped in 65 IV cases and 100 controls using Sequenom MassARRAY platform. Mass spectra of the two functional polymorphisms (7638G/G and 7717.9A/A) were loss of the A allele. Pausing and probe peaks are indicated above the graph.

Scotland and the USA [5]. Meanwhile, c.3321delA mutation in FLG were identified as prevalent in Japanese and Korean IV and AD patients [7,17]. FLG mutation E1795X was identified in the Taiwanese population [14]. Nonsense FLG mutations (p.S2889X and p.S3296X) were found in Japan [18]. Recent reports have suggested FLG mutations 1249insG, 7945delA, Q2147X E2422X and 441delA were found in Singaporean Chinese IV patients [15].

In our case-control study, we investigated 3321delA, 441delA, 1249 insG, E1795X and S3296X mutations which associated with Asian IV patients. With our results, we demonstrate here that more than $52.31 \%$ of IV patients in our Southern Chinese case series carry one FLG mutation 3321delA, whereas $4 \%$ of ethnically matched control individuals carry this mutation. Mutation 3321delA was firstly reported in a Japanese population [7], and was absent in the European population and Singaporean Chinese patients $[8,15]$. One mutation, 441-delA, was a previously unidentified FLG mutation which might be Singaporean Chinese specific [15]. FLG mutation 441delA was only found in one IV patients in our case-control study. This mutation may be less common in the Asian populations. FLG mutations 1249 insG, E1795X and S3296X were not identified either in patients with IV or in healthy controls. With our results, we could not only confirm the strong influence of the FLG mutations on the pathogenesis of the disease in this pop- ulation, but also exemplify differences in prevalent genetics of FLG mutations between Europe and Asia.

\section{REFERENCES}

[1] Wells, R.S. and Kerr, C.B. (1965) Genetic classification of ichthyosis. Archives of Dermatology, 92, 1-6.

[2] Anton-Lamprecht, I. and Hofbauer, M. (1972) Ultrastructural distinctive features of autosomal dominant ichthyosis vulgaris and X-linked recessive ichthyosis. Dermatologica, 145, 60-64.

[3] Sybert, V.P., Dale, B.A. and Holbrook, K.A. (1985) Ichthyosis vulgaris: Identification of a defect in synthesis of filaggrin correlated with an absence of keratohyaline granules. The Journal of investigative dermatology, 84, 191-194.

[4] Nirunsuksiri, W., Presland, R.B., Brumbaugh, S.G., Dale, B.A. and Fleckman, P. (1995) Decreased profilaggrin expression in ichthyosis vulgaris is a result of selectively impaired posttranscriptional control. The Journal of biological chemistry, 270, 871-876.

[5] Smith, F.J., Irvine, A.D., Terron-Kwiatkowski, A., Sandilands, A., Campbell, L.E. and Zhao, Y. (2006) Loss-offunction mutations in the gene encoding filaggrin cause ichthyosis vulgaris. Nature Genetics, 38, 337-342.

[6] Bremmer, S.F., Hanifin, J.M. and Simpson, E.L. (2008) Clinical detection of ichthyosis vulgaris in an atopic dermatitis clinic: Implications for allergic respiratory disease and prognosis. Journal of the American Academy of Dermatology, 59, 72-78.

[7] Nomura, T., et al. (2007) Unique mutations in the filaggrin gene in Japanese patients with ichthyosis vulgaris 
and atopic dermatitis. The Journal of Allergy and Clinical Immunology, 119, 434-440.

[8] Akiyama, M. (2010) FLG mutations in ichthyosis vulgaris and atopic eczema. Spectrum of mutations and population genetics. The British Journal of Dermatology, 162, 472-477.

[9] Sandilands, A., et al. (2006) Prevalent and rare mutations in the gene encoding filaggrin cause ichthyosis vulgaris and predispose individuals to atopic dermatitis. The Journal of Investigative Dermatology, 126, 1770-1775.

[10] Hamada, T., et al. (2008) De novo occurrence of the filaggrin mutation p.R501X with prevalent mutation c.3321delA in a Japanese family with ichthyosis vulgaris complicated by atopic dermatitis. The Journal of Investigative Dermatology, 128, 1323-1325.

[11] Sandilands, A., et al. (2007) Comprehensive analysis of the gene encoding filaggrin uncovers prevalent and rare mutations in ichthyosis vulgaris and atopic eczema. $\mathrm{Na}$ ture Genetics, 39, 650-654.

[12] Gruber, R., Janecke, A.R., Fauth, C., Utermann, G., Fritsch, P.O. and Schmuth, M. (2006) Filaggrin mutations p.R501X and c.2282del4 in ichthyosis vulgaris. European Journal of Human Genetics: EJHG, 15,179-184.
[13] Sinclair, C., et al. (2009) Filaggrin mutations are associated with ichthyosis vulgaris in the Bangladeshi population. The British Journal of Dermatology, 160, 1113-1115.

[14] Hsu, C.K., et al. (2009) Analysis of Taiwanese ichthyosis vulgaris families further demonstrates differences in FLG mutations between European and Asian populations. The British Journal of Dermatology, 161, 448-451.

[15] Chen, H., et al. (2008) Unique and recurrent mutations in the filaggrin gene in Singaporean Chinese patients with ichthyosis vulgaris. The Journal of Investigative Dermatology, 128, 1669-1675.

[16] Palmer, C.N., et al. (2006) Common lossof-function variants of the epidermal barrier protein filaggrin are a major predisposing factor for atopic dermatitis. Nature $\mathrm{Ge}$ netics, 38, 441-446.

[17] Kang, T.W., Lee, J.S., Oh, S.W. and Kim, S.C. (2009) Filaggrin mutation c.3321delA in a Korean patient with ichthyosis vulgaris and atopic dermatitis. Dermatology, 218, 186-187.

[18] Nomura, T., et al. (2008) Specific filaggrin mutations cause ichthyosis vulgaris and are significantly associated with atopic dermatitis in Japan. The Journal of Investigative Dermatology, 128, 1436-1441. 